\title{
Caracterización óptica de defectos superficiales en puntos cuánticos coloidales de Seleniuro de Cadmio (CdSe)
}

\author{
Optical characterization of surface defects in \\ cadmium selenide (CdSe) colloidal \\ quantum dots
}

Hamilton Alexander Ponce Elias, Carlos Ernesto Rudamas Flores

\begin{abstract}
Laboratorio de Espectroscopia Óptica, Escuela de Física, Facultad de Ciencias Naturales y Matemática, Universidad de El Salvador, Final 25 Av. Norte, San Salvador, América Central. e-mail: h.ponce3@gmail.com; carlos.rudamas@gmail.com
\end{abstract}

\section{RESUMEN}

En el presente estudio se han caracterizado ópticamente defectos superficiales ("non-stoichiometric" y "dangling bonds") en puntos cuánticos (QDs) coloidales de CdSe sintetizados por el método de descomposición térmica. En especial, se estudió la presencia de defectos vinculados con una alta relación área/volumen de los QDs. Los espectros de fotoluminiscencia (PL) de estos QDs presentan dos bandas, atribuibles a la recombinación excitónica y a la recombinación excitónica vía defectos superficiales. Luego de la síntesis las muestras permanecieron durante un tiempo sin ningún tratamiento adicional. Durante este tiempo se observó mediante el monitoreo de la evolución de las bandas de PL, un incremento en la intensidad de la banda atribuible a defectos respecto a la de emisión excitónica. Posteriormente, las muestras se lavaron utilizando abundante etanol y fueron disueltas en 1-octadeceno. Con este tratamiento se ha observado una recuperación gradual de la PL de la banda excitónica. Esto podría atribuirse a la remoción de los residuos de la reacción que pudieron formar defectos y a un reacomodo de los surfactantes, mostrando de esta manera una forma de pasivación de defectos superficiales. Para todas las muestras estudiadas en este trabajo, se observó mayor presencia de defectos superficiales para QDs con mayor relación área/volumen.

Palabras clave: Fotoluminiscencia, puntos cuánticos coloidales, CdSe, defectos superficiales, surfactantes.

\begin{abstract}
In this work, non-stoichiometric defects and dangling bond defects were optically characterized for CdSe colloidal quantum dots (QDs), which were prepared by the thermal decomposition method. Particularly, the presence of defects linked to a high QD surface to volume ratio was studied. The photoluminescence (PL) spectra of the samples show two bands, which could be associated to excitonic recombination and excitonic recombination via surface defects. The samples remained untreated for a period of time after preparation. During this time a gradual deterioration of the QDs was observed by monitoring the evolution of PL spectra. An increase in intensity of the band associated to recombination via defects, with respect to the excitonic emission band, was observed. Subsequently, the samples were washed using abundant ethanol and dissolved in 1-octadecene. After this treatment a gradual recovery of the excitonic PL band was observed. This could be attributed to the removal of reaction residues that could give rise to defects, and to a rearrangement of the surfactants, thus showing a form of surface defects passivation. For all the samples, investigated in this work, a greater presence of surface defects for QDs of higher surface to volume ratio was observed.
\end{abstract}

Keywords: Photoluminescence, colloidal quantum dots, CdSe, surface defects, surfactants.

\section{INTRODUCCIÓN}

En las últimas décadas se ha tenido un gran interés por el estudio de QDs en diversas disciplinas (medicina, física, biología y química) debido a sus excelentes propiedades de sintonización, funcionalización y emisión de luz, entre otras $[\underline{1}, \underline{2}]$. Dada las dimensiones nanométricas que poseen, estos tienen una alta relación 
área/volumen que resulta en una fracción grande de átomos superficiales en relación con el total de átomos del cristal, pudiendo dar lugar a la formación de sitios de defectos no homogéneos en la superficie. Éstos son capaces de atrapar electrones e impedir la recombinación radiativa, degradando de esta forma la eficiencia cuántica de las estructuras [3,4]. Se ha demostrado también que los defectos superficiales en QDs afectan la eficiencia de conversión fotovoltaica [5-7]. En la actualidad se han desarrollado dos formas importantes de pasivación de defectos superficiales en QDs sin envolvente (Core). Por una parte la orgánica la cual depende de los surfactantes empleados para estabilizar los QDs [5] y por otra parte la atómica, que depende de la introducción de iones haluros en la superficie de los QDs y ha mostrado mejoras en la conducción de estos [6,7]. También ha sido empleada por algunos autores una pasivación hibrida que emplea ambas formas de pasivación simultáneamente [8]. Por lo anteriormente expuesto, en el presente trabajo se ha desarrollado una caracterización óptica de defectos superficiales ("non-stoichiometric" y "dangling bonds") en QDs de CdSe y se ha investigado el comportamiento temporal de dichos sitios de defectos utilizando espectroscopia de fotoluminiscencia (PL). También se ha estudiado la dependencia de la presencia de defectos superficiales luminiscentes con la relación área/volumen y la pasivación de éstos mediante un método de lavado, que se propone como otra vía de pasivación de defectos superficiales para QDs degradados.

\section{MATERIALES Y MÉTODOS}

\subsection{Síntesis}

Los QDs se sintetizaron a partir de dos soluciones, utilizando un método similar al propuesto por BOATMAN et al. [9]. Una solución precursora (A) de selenio se preparó en un matraz con la adición de los siguientes componentes: $30 \mathrm{mg}$ de selenio, $8 \mathrm{ml}$ de 1-octadeceno (ODE) y $0.4 \mathrm{ml}$ de trioctilfosfina. Se calentó a $225^{\circ}$ $\mathrm{C}$ y se dejó reposar. La segunda solución precursora (B) se preparó inmediatamente después de la solución A, en un matraz de $25 \mathrm{ml}$ con la adición de: $13 \mathrm{mg}$ de óxido de cadmio (CdO), $0.6 \mathrm{ml}$ de ácido oleico (AO) y 8 $\mathrm{ml}$ de 1-Octadeceno. Esta solución se llevó a $225^{\circ} \mathrm{C}$. La solución A se depositó en el matraz de la solución B, manteniendo la misma temperatura, inmediatamente se extrajeron muestras de la solución resultante y se depositaron en tubos de ensayo, colocando inmediatamente estos en hielo con el fin de cortar la reacción y posteriormente se etiquetaron de M1 a M7 en el siguiente orden temporal: 2 segundos para M1, 12 segundos para M2, 22 segundos para M3, 32 segundos para M4, 42 segundos para M5, 62 segundos para M6 y 82 segundos para M7. Los tamaños promedios de los QDs han sido estimados utilizando la aproximación de la masa efectiva considerando una geometría esférica, requiriendo para dicho cálculo los máximos de las bandas de absorción atribuible a la primera transición excitónica [10]. Los radios así estimados para los QDs investigados en este trabajo se encuentran entre $1.73 \mathrm{~nm}$ para M1 y $2.33 \mathrm{~nm}$ para M7 [11]. Para estos radios las relaciones áreas/volumen varían desde $1.7 \mathrm{~nm}^{-1}$ para M1 hasta $1.2 \mathrm{~nm}^{-1}$ para M7.

\subsection{Método de lavado}

Las muestras de QDs se lavaron según el método descrito por ZEZZA et al. [12] con la diferencia que en este trabajó se aplicó a QDs envejecidos o degradados. Se añadió etanol en exceso, se agitó y centrifugó la solución resultante y se procedió a separar el sobrenadante. Posteriormente se agregó nuevamente abundante etanol repitiendo 8 veces el proceso antes descrito. Cuando se retiró por última vez el sobrenadante se procedió a medir los espectros de PL para las diferentes concentraciones de ODE agregadas, como se enumera a continuación: $0 \mathrm{ml}, 0.1 \mathrm{ml}, 0.2 \mathrm{ml}, 0.3 \mathrm{ml}, 0.4 \mathrm{ml}, 0.5 \mathrm{ml}, 0.6 \mathrm{ml}, 0.7 \mathrm{ml}, 0.8 \mathrm{ml}, 0.9 \mathrm{ml}$ y $1.0 \mathrm{ml}$.

\subsection{Montaje experimental}

Para la toma de espectros de PL, se utilizó el montaje experimental cuyo esquema se muestra en la Fig. 1. El mismo consta de los siguientes componentes: una fuente de luz (FL), dos lentes colimadores (L1 y L2), un filtro BP35 (F), muestras de QDs (M), fibras ópticas (FO), un espectrógrafo (E) y una computadora (PC).

En el presente trabajo, se utilizaron dos espectrógrafos: USB4000 -UV-VIS (usado para medir los espectros de la Fig. 2) y USB4000-FL (para los espectros de las Figs. 3, 4, y 5) de la Firma Ocean Optics con una resolución espectral de $1.5 \mathrm{~nm}$ y $10 \mathrm{~nm}$, respectivamente. Como fuente de luz se utilizó una lámpara de mercurio. Con el filtro BP35 se seleccionó la línea del mercurio ubicada a $365 \mathrm{~nm}$, que se utilizó como fuente de excitación. 


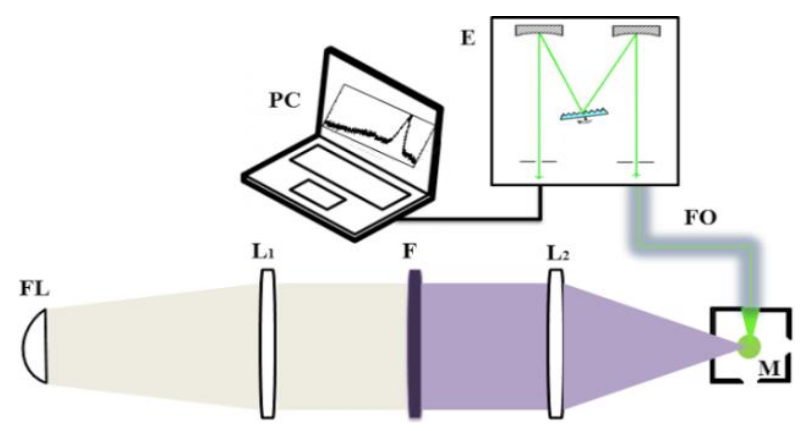

Figura 1: Montaje experimental de fotoluminiscencia.

\section{RESULTADOS}

\subsection{Degradación de puntos cuánticos}

Posterior a la síntesis, los QDs se mantuvieron por un tiempo sin ningún procedimiento adicional. Durante este tiempo, se monitorearon por medio de fotoluminiscencia, con el fin de observar su degradación.

En todos los espectros medidos para las muestras en estudio, se observaron dos bandas, una a menores longitudes de onda y una ancha a mayores longitudes de onda. Estas bandas son atribuibles a la emisión debido a la recombinación excitónica y a la emisión debido a la recombinación excitónica vía defectos superficiales, respectivamente. Otros autores han observado también bandas similares a éstas [13,14]. En el presente trabajo estas dos bandas se denominarán por simplicidad banda excitónica y banda de defectos superficiales. Para la muestra M4, dichas bandas poseen máximos aproximadamente en 508 nm y 637 nm, (véase Fig. 2(a)). En dicha figura se presentan espectros de PL de las muestras M1 a M7, normalizados al máximo excitónico. En la Fig. 2(b) se muestran espectros de PL para M4, tomados en un intervalo temporal de 6 meses. En estos espectros de PL, la banda atribuible a defectos superficiales incrementa en intensidad, relativa a la banda excitónica, al aumentar el tiempo de envejecimiento.

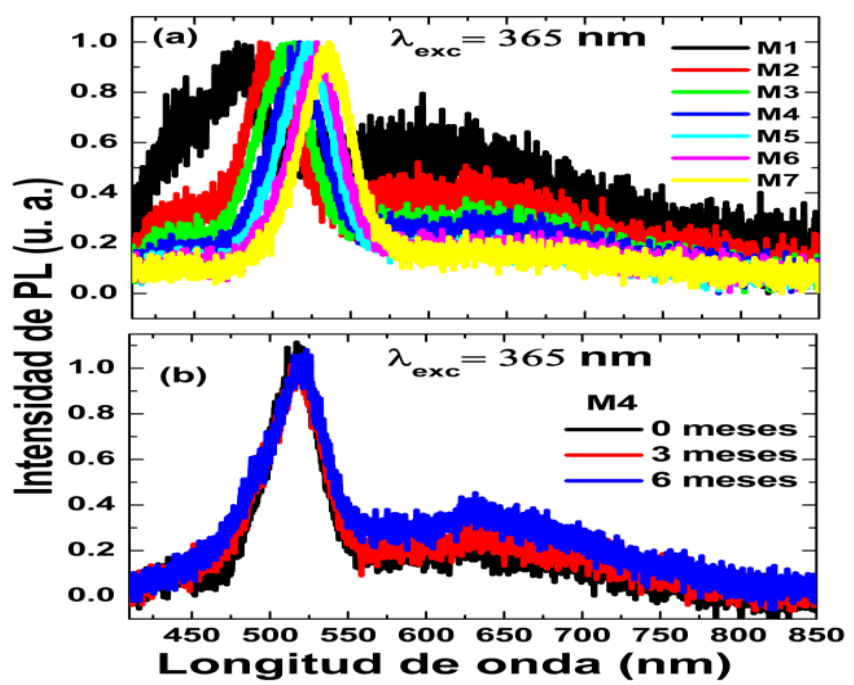

Figura 2: (a) Espectros de PL de las 7 muestras de QDs a dos días de la síntesis; (b) Espectros de PL para la muestra M4 en los primeros 6 meses.

\subsection{Influencia del lavado en la pasivación de defectos}

En la Fig. 3, se muestra una secuencia de espectros de PL para M1, tomados al agregar 1-octadeceno en una serie de $0.1 \mathrm{ml}$ del mismo después del lavado con etanol, como se describió en la sección 2.2. En dicha figura se puede observar que la banda ancha atribuida a los defectos superficiales disminuye considerablemente en relación a la banda excitónica, a medida aumenta la concentración del ODE. 


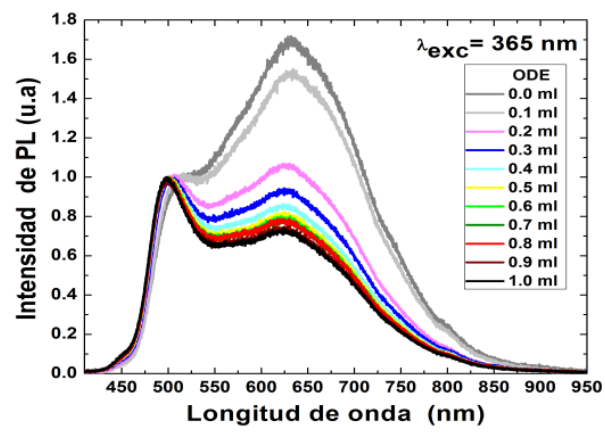

Figura 3: Espectros de PL normalizados al máximo excitónico, para la muestra M1 después del lavado para diferentes concentraciones de ODE.

Se observó también que después de agregar $1 \mathrm{ml}$ de ODE y dejar reposar, la banda atribuible a defectos superficiales en los espectros de PL sigue disminuyendo, en relación a la banda atribuible a excitones, como se muestra en la Fig. 4.

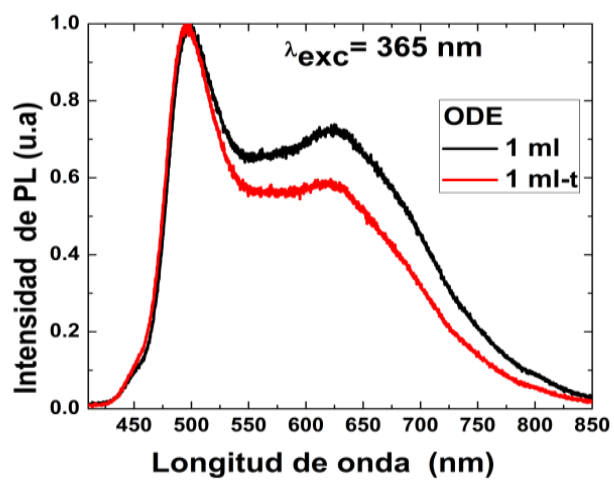

Figura 4: Espectros de PL normalizados al máximo excitónico para la muestra M1, después del lavado. Línea negra, al agregar $1 \mathrm{ml}$ de ODE. Línea roja, medido 15 días después de agregado $1 \mathrm{ml}$ de ODE.

En este estudio también se ha observado que el método de lavado descrito en la Sección 2.2 aplicado a QDs degradados, proporciona una vía de pasivación de defectos superficiales que vuelve la intensidad de la banda de emisión de estos últimos, respecto al máximo de la banda excitónica, muy similar a la intensidad de la banda de emisión de defectos superficiales de los QDs recién preparados. Esto es evidente si se comparan los espectros para la muestra M1 de las Figs. 5(a) y 5(b) que representan los QDs degradados antes y después del lavado con el espectro para M1 recién preparada de la Fig.2 (a).

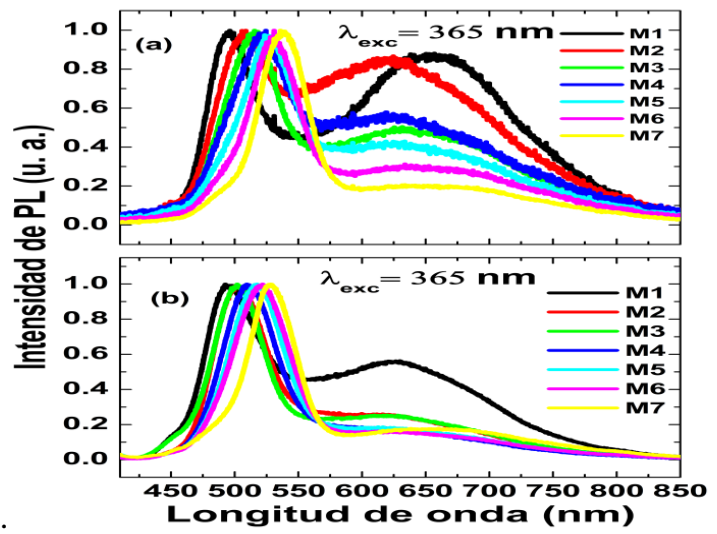

Figura 5: Espectros de PL de las muestras M1 a M7 estudiadas (a) dos años después de la síntesis (b) después del lavado y disueltos en $1 \mathrm{ml}$ de 1-octadeceno. 


\section{DISCUSIÓN}

En la Fig. 2(a), se puede observar un desplazamiento hacia el azul de la banda excitónica en los espectros de PL de las muestras recién sintetizadas, cuando el tamaño de los QDs disminuye. Este desplazamiento se asocia al efecto de confinamiento cuántico, el cual ha sido también observado por otros autores [1,2]. También se observa que a medida que aumenta el tamaño de las nanoestructuras, la banda ancha atribuible a los defectos superficiales disminuye en relación con la banda atribuible a excitones. Esta tendencia podría estar relacionada con la disminución de la relación área/volumen de $1.7 \mathrm{~nm}^{-1}$ para M1 hasta $1.2 \mathrm{~nm}^{-1}$ para M7, ya que cuando el tamaño de las nanoestructuras aumenta existe una fracción menor de átomos superficiales relativa a los átomos totales de la estructura, lo que está de acuerdo con lo descrito por SILVA et al. [15]. En la Fig. 2(b), se presentan espectros de PL para M4, tomados a 0, 3 y 6 meses de envejecimiento. Se observa un incremento en intensidad relativa de la banda atribuible a defectos superficiales respecto al máximo excitónico, al aumentar el tiempo de envejecimiento de los QDs. Eso es atribuido al efecto de envejecimiento de QDs [16-18], mostrando una mayor presencia de defectos superficiales respecto a espectros de PL en muestras recién sintetizadas.

En la Fig. 3 de la sección 3.2, se mostró que la banda atribuible a defectos superficiales posterior al lavado, disminuyó en relación al máximo excitónico al agregar ODE. Este fenómeno, se puede entender teniendo en cuenta que en ausencia de 1-octadeceno el ácido oleico y trioctilfosfina (surfactantes) tienen mayor desorción de la superficie de las estructuras, lo que hace más evidentes los efectos de los defectos superficiales sobre la PL de los QDs, como ha sido discutido por otros autores [4]. Al agregar ODE, este podría aumentar la adsorción de los surfactantes, pasivando de esta forma los defectos superficiales. Dicha pasivación de defectos superficiales explicaría la disminución de la intensidad de la banda de emisión atribuible a defectos respecto a la banda excitónica, la cual aumenta su intensidad de emisión.

En la Fig. 4, La disminución en intensidad de la banda atribuible a defectos respecto a la banda excitónica después de haber agregado ODE y dejar reposar por un tiempo ya ha sido reportado por ZEZZA et al. [12] y se asocia al reacomodo superficial de los surfactantes que se da posterior al lavado.

Por último en la Fig. 5 se puede observar que lavar con etanol y agregar ODE a muestras de QDs degradados representó una forma de pasivación de defectos superficiales, que vuelve la intensidad de la banda de emisión atribuible a estados superficiales, respecto al máximo de la banda excitónica, muy similar a la intensidad de la banda de emisión de defectos superficiales de los QDs recién preparados. Esto se puede observar comparando los espectros de PL antes del lavado, Fig. 5(a), con los espectros de PL después del lavado, Fig. 5(b). Es evidente la disminución en intensidad de la banda atribuible a defectos superficiales respecto a la banda excitónica. Esto podría atribuirse a la remoción de los residuos de la reacción que pudieron formar defectos y un reacomodo de los surfactantes como se describió en el párrafo anterior, mostrando de esta manera una forma de pasivación de defectos superficiales en QDs envejecidos. Es de hacer notar que el método de lavado empleado en este trabajo, en contraste con el descrito por ZEZZA et al. [12], el cual es aplicado a muestras "recién" sintetizadas, es aplicado para QDs envejecidos. Los resultados obtenidos mediante este método muestran una mayor pasivación para muestras con una mayor relación área/volumen, es decir, QDs de menor tamaño.

\section{CONCLUSIONES}

En el presente trabajo se han caracterizado QDs de CdSe por medio de espectroscopia de PL. Se ha observado que las muestras sufren degradación al transcurrir el tiempo. Esta degradación se vuelve evidente en el incremento de la intensidad de la banda atribuible a defectos superficiales respecto a la banda excitónica. Para todas las muestras investigadas en el presente estudio, se observa mayor presencia de defectos superficiales para QDs de mayor relación área/volumen. También se ha observado que el método de lavado aplicado en este trabajo a QDs envejecidos y degradados, se podría considerar como una forma eficiente para pasivar defectos superficiales de QDs en las condiciones antes mencionadas. Esta pasivación se vuelve más importante para QDs de mayor relación área/volumen.

\section{AGRADECIMIENTOS}

Al Consejo de Investigaciones Científicas de la Universidad de El Salvador (CIC-UES) por el financiamiento parcial a esta investigación bajo el proyecto 09.20 y a M. Pacheco por el financiamiento parcial en la compra de reactivos. También se agradece a M. Barrios y D. Pleitez por las fructíferas discusiones durante la preparación de este trabajo. 


\section{BIBLIOGRAFÍA}

[1] JUÁREZ, B.H., "Nanopartículas semiconductoras coloidales y aplicaciones”, Anales de Química, v. 107, n. 3, pp. 229-236, Mzo. 2011.

[2] POMPO, V., GOYANES, V., "Puntos cuánticos: Nueva aportación de la nanotecnología en investigación y medicina", Revista Complutense de Ciencias Veterinarias, v. 5, n. 1, pp. 69-102, En. 2011.

[3] BABENTSOV, V. N., " Defects with deep donor and acceptor levels in nanocrystals of CdTe and CdSe" Semiconductor Physics, Quantum Electronics \& Optoelectronics, v. 9, n. 3, pp. 94-98, Oct. 2006

[4] YOST, J., Synthesis and Surface Modification of CdSe and CdS Quantum Dots Exhibiting High Quantum Yield,Thesis M.Sc., Bucknell University, Lewisburg, Estados Unidos, 2012.

[5] TANG, J., KEMP, W., HOOGLAND, S., et al., "Colloidal-quantum-dot photovoltaics usingatomicligand passivation”, Nature Materials, v. 10, 765-771, Sep. 2011.

[6] ZANELLA, M., MASERATI, L., LEAL, M.P., et al, "Atomic Ligand Passivation of Colloidal Nanocrystal Films via their Reaction with Propyltrichlorosilane” Chemistry of Materials, v. 25, n. 8, 1423-1429, En. 2013.

[7] HEO, S.J., YOON, S., OH, S.H., et al., "Modification of hybrid active bilayer for enhanced efficiency and stability in planar heterojunction colloidal quantum dot photovoltaics", Nanoscale Research Letters, v. 8:488, n. 1, Nov. 2013.

[8] IP, A. H., THON, S. M., HOOGLAND, S., et al., "Hybrid passivated colloidal quantum dot solids", $N a$ ture Nanotechnology, v. 7, 577-582, Sep.2012.

[9] BOATMAN, E. M., LISENSKY, G. C., NORDELL, K. J., et al, “A Safer, Easier, Faster Synthesis for CdSe Quantum Dot Nanocrystals”, Journal of Chemical Education, v. 82, n. 11, pp. 1697-1699, Nov. 2005.

[10] MANSUR, H. E., MANSUR, A. A. P., "CdSe quantum dots stabilized by carboxylic-functionalized PVA: Synthesis and UV-vis spectroscopy characterization”, Materials Chemistry and Physics, v. 125, 709717, Sep. 2010.

[11] PACHECO, M. y RUDAMAS, C., "Fabrication and optical characterization of CdSe quantum Dots", In. $62^{\text {nd }}$ Annual Meeting of the Austrian Physical Society, Ed. M. Ramsey, pp. 159, Graz, Austria, Sep. 2012. [12] ZEZZA, F., COMPARELLI, R., STRICCOLI, M., et al, "High quality CdS nanocrystals: surface effects", Synthetic Metals, v. 139, pp. 597-600, Oct. 2003.

[13] KIM, J. Y., VOZNYY, O., ZHITOMIRSKY, D., et al. "25th Anniversary Article: Colloidal Quantum Dot Materials and Devices: A Quarter-Century of Advances”, Advanced Materials, v. 25, pp. 4986-5010, Ene. 2013.

[14] WANG, L. W., y ZUNGER, A., "Pseudopotential calculations of nanoscale CdSe quantum dots", Physical Review B, v. 53, n. 15, 9579-9582 Abr. 1996.

[15] DA SILVA, R. S., NETO, E. S., DANTAS, N. O. “Optical, Magnetic, and Structural Properties of Semiconductor and Semimagnetic Nanocrystals", In: Nanocrystals-Synthesis, Characterization and Applications, v. 1, Janeza Trdine 9, 5100 Rijeka. Croacia, pp. 63-80, 2012.

[16] ALIVISATOS, A. P., "Perspectives on the Physical Chemistry of Semiconductor Nanocrystals", Journal of Physical Chemistry, v. 100, pp. 13226-13239, Mar. 1996.

[17] CORDERO, S. R., CARSON, P. J., ESTABROOK, R. A., et al. "Photo-Activated Luminescence of CdSe Quantum Dot Monolayers", Journal of Physical Chemistry B, v. 104, pp. 12137-12142, Agos. 2000.

[18] BHATTACHARJEE, B., HSU, C. H., LUA, C. H., et al, “Aging Behaviour of CdSe Nanoparticles: Effect of Stoichiometry on Changing Physical Properties", Chalcogenide Letters, v. 7, n. 1, pp. 71-81, Ene. 2010 . 